\title{
A single-crystalline silver plasmonic circuit for visible quantum emitters
}

\author{
Christian Schörner ${ }^{\dagger}$, Subhasis Adhikari ${ }^{\dagger}$, Markus Lippitz ${ }^{\dagger}, *$ \\ $\dagger$ Experimental Physics III, University of Bayreuth, Universitätsstr. 30, 95440 Bayreuth, Germany \\ Corresponding Author \\ * Email: markus.lippitz@uni-bayreuth.de
}

\section{table of content}

Figure S1: Energy dispersive X-ray spectroscopy of silver plates

Figure S2: Electron backscatter diffraction analysis of silver plates

Figure S3: Atomic force microscopy of silver plates

Figure S4: Protection of silver plates

Figure S5: Laser spot size

Figure S6: Mode detector images at all wavelengths

Figure S7: Incoupling, propagation and detection efficiencies

Figure S8: Simulation of the beating pattern

Figure S9: Emission spectra along the waveguide 

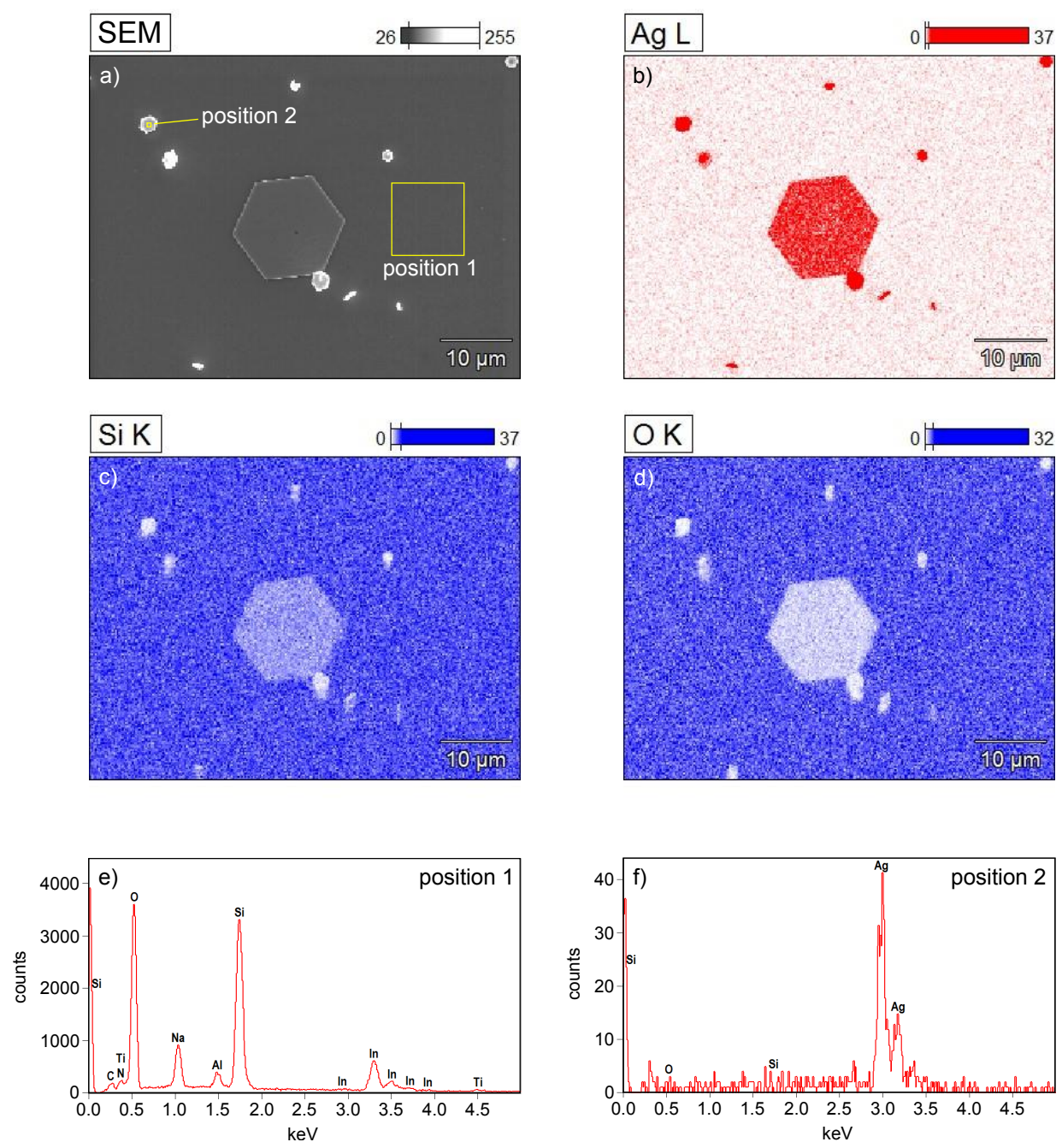

Figure S1: Energy-dispersive X-Ray analysis of silver plates on a borosilicate glass substrate coated with $40 \mathrm{~nm}$ of ITO. (a) Scanning electron micrograph of the investigated area. (b-d) Colormaps of the $\mathrm{Ag}$, Si and O content of area shown in (a). The silver plates can be identified by a high content of silver and lower content of Si and O. Due to the penetration depth of the electrons of several hundreds of nanometers, the EDX signal for thin Ag plates also features content from the ITO substrate below (e.g. Si and O, see big Ag plate in the middle). (e) EDX-spectrum of an empty position on the ITO substrate (position 1, size $10 \times 10 \mu \mathrm{m}$, c.f. a) yielding approximately $40 \% \mathrm{O}$, $20 \% \mathrm{Si}, 30 \% \mathrm{In}$ and small contents of other elements. (f) EDX-spectrum from a small Ag plate (position 2, size $0.5 \times 0.5 \mu \mathrm{m}$, c.f. a) shows nearly only silver content (very low $\mathrm{Si}$ and O content). Due to the small lateral size of the plate, i.e. high thickness (see AFM data in figure S3c), the EDX signal predominantly steams from the Ag plate in this case. 

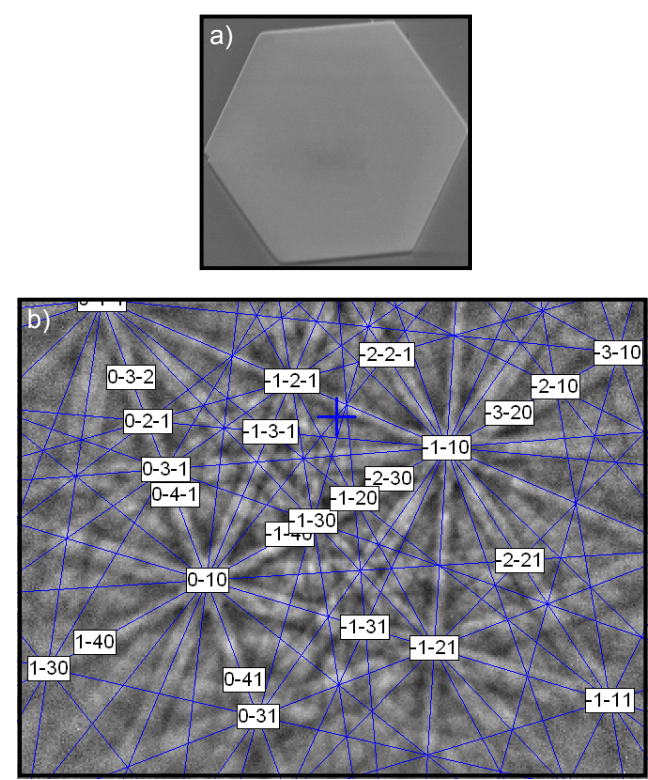
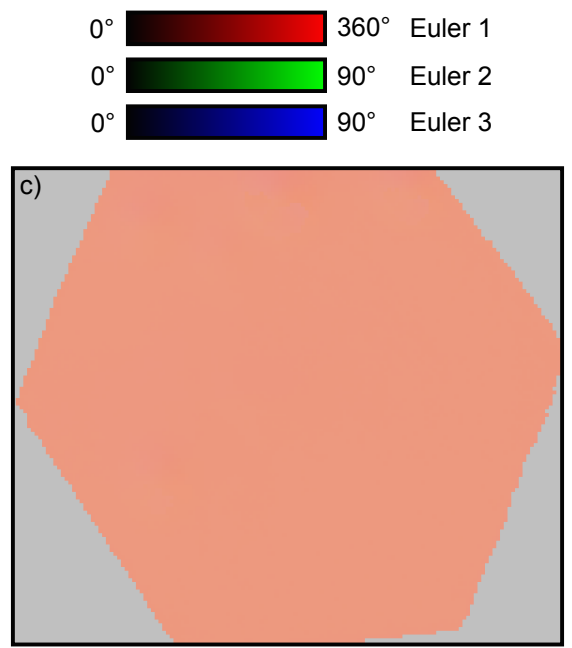

d)

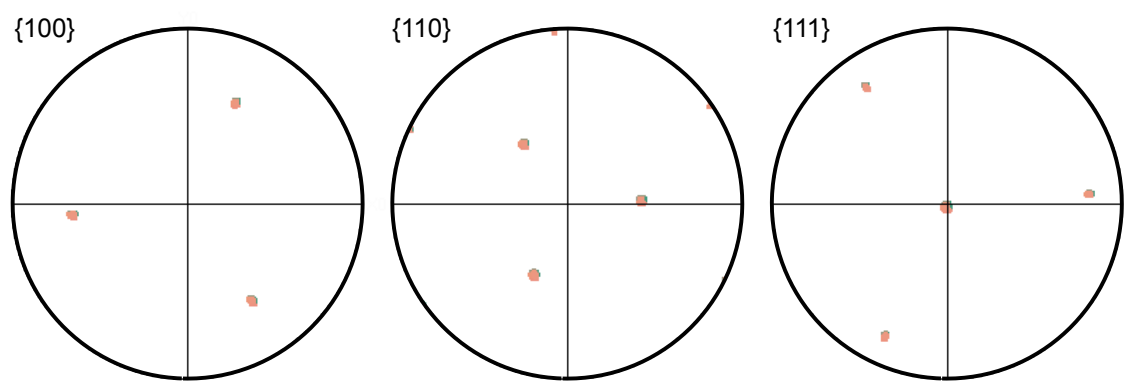

Figure S2: Electron backscatter diffraction (EBSD) analysis of silver plates. (a) Scanning electron micrograph of the investigated hexagonal Ag plate. (b) EBSD pattern detected at a single position on the plate. Kikuchi lines are indexed with the corresponding reciprocal lattice vector indices. Kikuchi band intersections are labeled with the corresponding direct lattice indices. (c) Euler map obtained from scanning across the plate and using the obtained Euler angles as color-channels for the Euler map representation. $120^{\circ}$ shifts of Euler angle 1 are equivalent due to the three-fold symmetry along the (111) plane and have been corrected to yield the uniform color representation. (d) Pole figures, i.e. stereographic projection of the directional distribution of all crystallographically equivalent lattice vectors. 

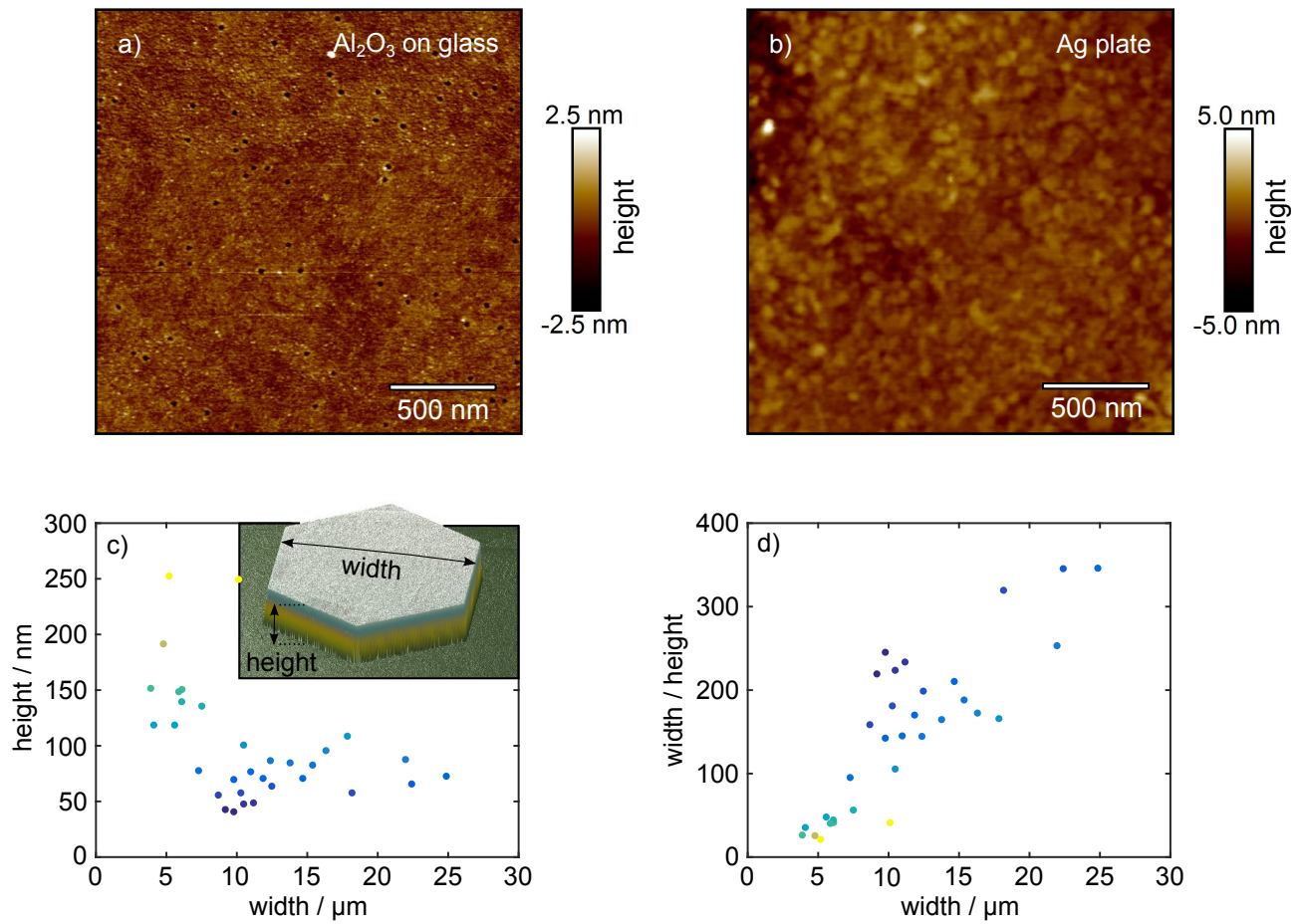

Figure S3: (a) Atomic force microscope (AFM) topography scan of a $\mathrm{Al}_{2} \mathrm{O}_{3}$ layer deposited on glass by atomic layer depostion (125 cycles). (b) AFM topography scan of the surface of an Ag plate (without $\mathrm{Al}_{2} \mathrm{O}_{3}$ layer). (c) The height of the silver plates as a function of the width. The height is measured between the glass surface and the top surface of the plate. The width is measured between opposite edges of the hexagonal shape (see inset). (d) The aspect ratio (width divided by height) as function of the plate's width. 

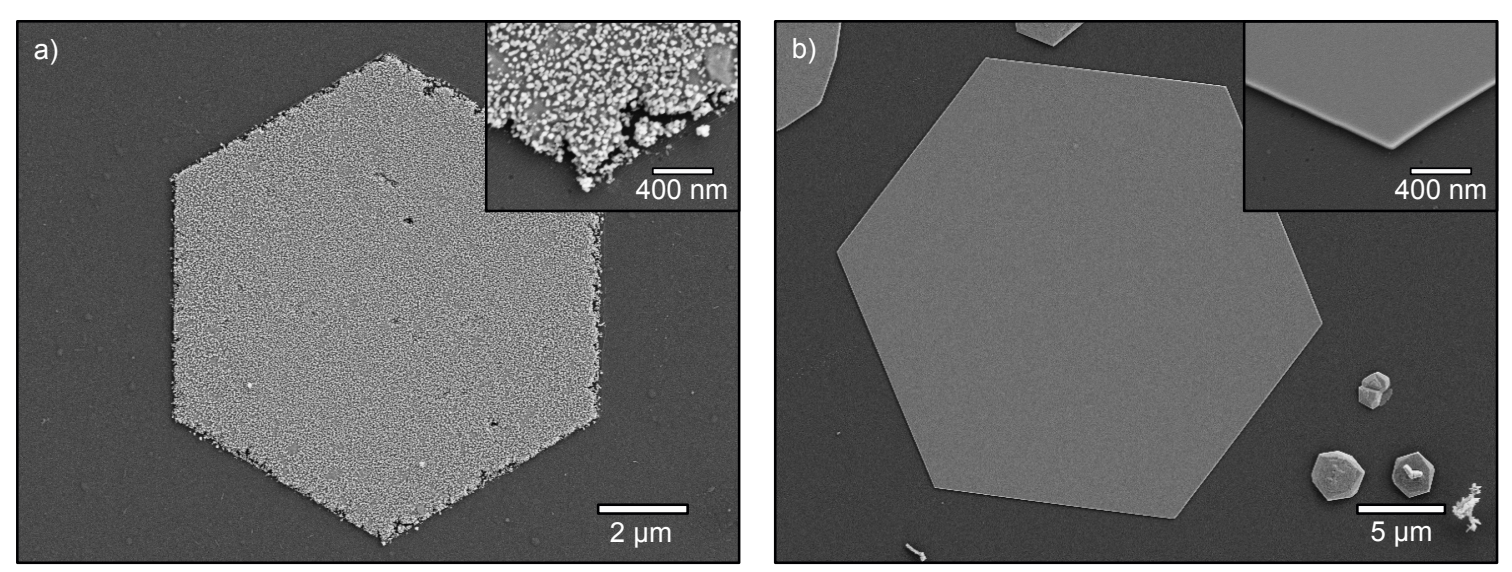

Figure S4: (a) Scanning electron microscope (SEM) image of a non-protected silver plate on glass. (b) SEM image of a silver plate on glass protected by a thin layer of $\mathrm{Al}_{2} \mathrm{O}_{3}$ deposited by atomic layer deposition (125 cycles, trimethylaluminium and water precursors, $80^{\circ}$ deposition temperature, about $10 \mathrm{~nm}$ thickness). The images have been taken after 6 weeks. During that time the samples have been stored at ambient conditions and exposed several times to acetone, iso-propanol and water. We conclude, that a thin layer of $\mathrm{Al}_{2} \mathrm{O}_{3}$ can protect the silver reasonably well from air and chemical treatments. 

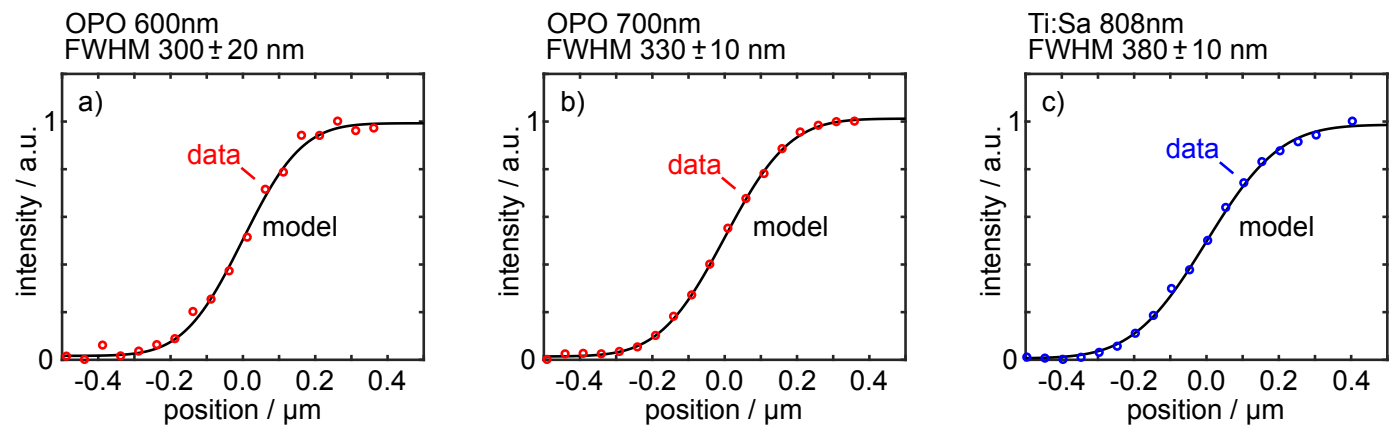

Figure S5: Laser spot sizes measured by scanning the laser focus across an edge of a silver plate and recording the reflected intensity. The experimental data is shown as colored circles while the fit of an error-function is shown as black solid line. The full width at half maximum of the laser focus is calculated from the width of the error-function. (a) Optical parametric oscillator (OPO) visible output at $600 \mathrm{~nm}$. (b) OPO visible output at $700 \mathrm{~nm}$. (c) Ti:Sa at $808 \mathrm{~nm}$. 

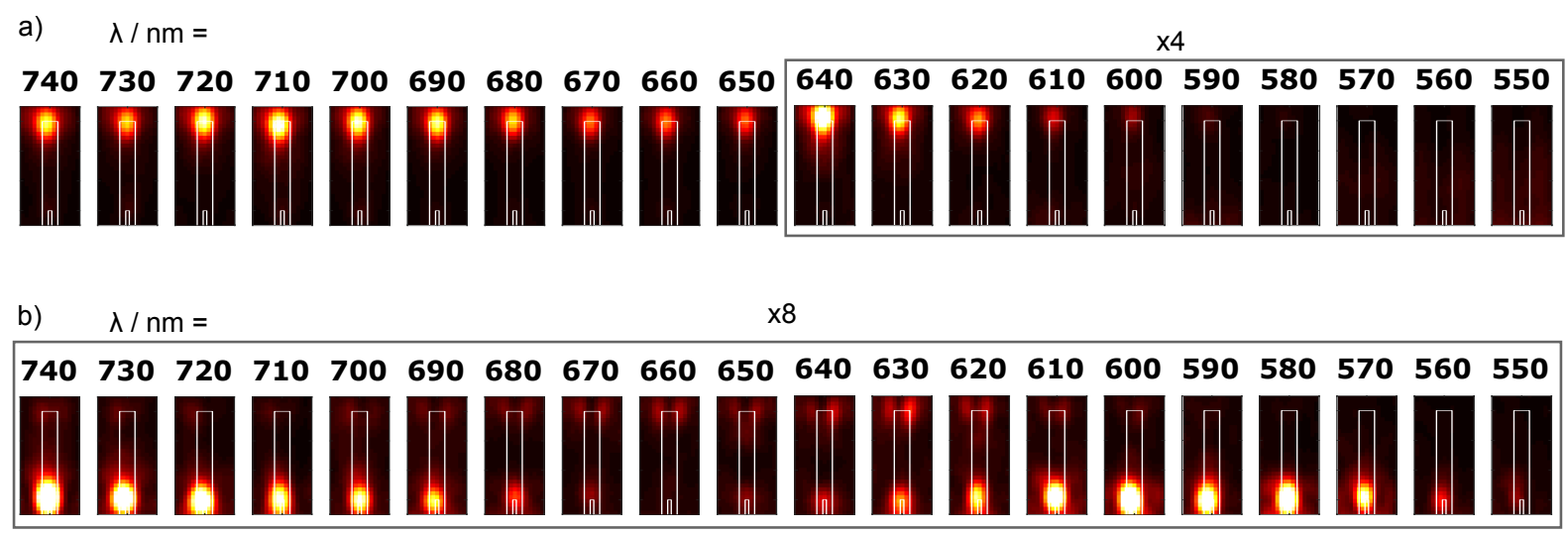

Figure S6: (a) Wavelength-dependent mode detector signals upon exciting the antenna with parallel polarized light (with respect to the two-wire waveguide). For better visibility, the data from 640 $550 \mathrm{~nm}$ is multiplied by a factor of 4 . (b) Mode detector signals upon exciting the antenna with perpendicular polarized light. Here, the signals are multiplied by a factor of 8 as compared to (a). We observe an oscillation between strong signals at the termination of the gap (e.g. $740 \mathrm{~nm}$ ) to a two-lobed emission at the far end (e.g. $660 \mathrm{~nm}$ ) and back again to a single lobed emission at the gap-termination (e.g. $600 \mathrm{~nm}$ ). 

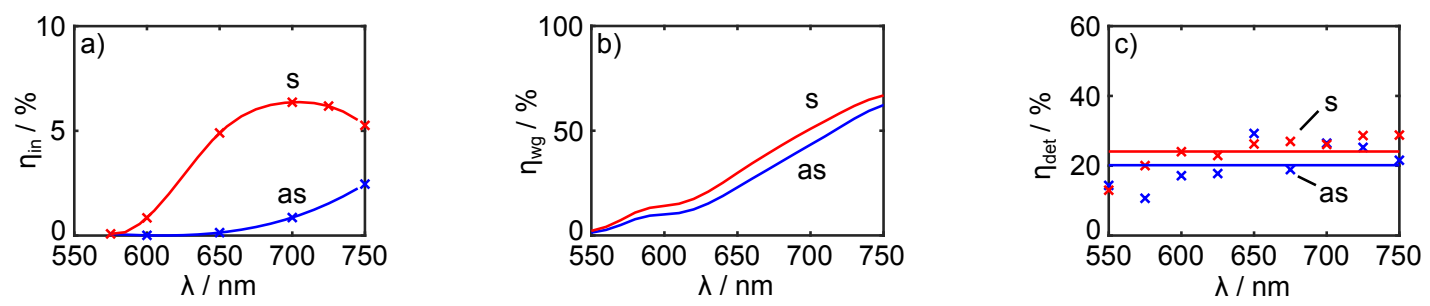

Figure S7: (a) Simulated incoupling efficiencies of the antenna. Crosses represent simulated values, while lines represent a spline-interpolation as a guide to the eye. (b) Calculated propagation efficiencies of the two-wire waveguide. (c) Calculated detection efficiencies of the mode detector. Crosses represent simulates values, while lines indicate the average values. In (a)-(c) the case of the symmetric (s) and anti-symmetric (as) mode is shown in red and blue, respectively.

\section{Incoupling efficiencies}

We define the incoupling efficiency of the optical antenna as the launched modal power of the symmetric (anti-symmetric) waveguide mode normalized to the power $\mathrm{P}_{0}$ of an incident Gaussian beam of parallel (perpendicular) polarization with respect to the waveguide. The Gaussian is focused on the antenna's center from the substrate's side with normal incidence and a full width at half maximum of half of the free-space wavelength $\lambda_{0}$.

In a 3D numerical Comsol model the modal power is evaluated at a port after about $l=2 \mu \mathrm{m}$ of the two-wire waveguide, well separated by the excitation position on the antenna, by the following equation:

$$
\eta_{i n, i}=e^{\left(4 \pi l \cdot\left|\operatorname{Im}\left(n_{e f f, i}\right)\right| / \lambda_{0}\right)} \int S_{\perp} d A \frac{\left|\int \vec{E} \cdot \vec{E}_{m o d e}^{*} d A\right|^{2}}{\int\left|\vec{E}_{\text {mode }}\right|^{2} \mathrm{~d} A \cdot \int|\vec{E}|^{2} d A} / P_{0}
$$

The integration of the normal component of pointing vector $S_{\perp}$ gives the total power outflow through the port area. The multiplication with the squared mode-overlap integral between the total field $\vec{E}$ at the port and the modal field $\vec{E}_{\text {mode, } i}$ results in the power outflow through the port carried by the waveguide mode. The integrations are performed over the port area. The parameter $i$ distinguishes between the case of the symmetric or anti-symmetric mode. The prefactor corrects the propagation losses toward the mode port, using the known imaginary part of the mode index $\operatorname{Im}\left(n_{e f f, i}\right)$.

The cross-section of the two-wire part is like the one shown in figure 1 of the main manuscript. The antenna is modelled as two cuboids with each having a length of $180 \mathrm{~nm}$, width of $90 \mathrm{~nm}$ and height of $40 \mathrm{~nm}$ (c.f. SEM-image in figure 3a of the main manuscript) attached to the incoupling end of the two-wires.

The computed values for the incoupling efficiencies are given in figure S7a.

\section{Propagation efficiencies}

The propagation efficiencies $\eta_{w g, i}$ for the symmetric and anti-symmetric mode along the two-wire waveguide part with a length of $l=3.5 \mu \mathrm{m}$ (figure S7b) are calculated according to:

$$
\eta_{w g, i}=\exp \left(-l / l_{p r o p, i}\right)
$$


The propagation lengths $l_{\text {prop }, i}$ are shown in figure 1 of the main manuscript.

\section{Detection efficiencies}

We define the detection efficiency of the mode detector for the symmetric (anti-symmetric) mode as the collected power at the far (near) end of the mode detector normalized to the modal power at the junction with the two-wire waveguide.

In a 3D numerical simulation a two-wire waveguide mode is launched by a mode port and propagating toward a mode detector. We evaluate the power flow through a sphere of $300 \mathrm{~nm}$ radius, centered at the mentioned mode detector position. The normal component of the pointing vector is integrated on the sphere area corresponding to the solid angle of our objective of $N A=1.35$ on the substrate's side. We correct this power by the small propagation losses of the two-wire part and normalize it to the applied power at the mode port. The propagation losses along the mode detector for detecting the symmetric mode at the far end of the mode detector are included in the detection efficiency of the symmetric mode.

The two-wire cross-section is equivalent to that of the main manuscript. For modeling the $1.5 \mu \mathrm{m}$ long mode detector, the gap is closed and filled with silver. We neglect the $\sim 5 \mathrm{~nm} \mathrm{Al}_{2} \mathrm{O}_{3}$ layer in the 3D simulation for numerical simplicity of this 3D model.

We find that the detection efficiencies are spectrally flat across the considered wavelength range. The average value for the symmetric and anti-symmetric modes are $\eta_{\mathrm{det}, i}=25 \%$ and $20 \%$, respectively (figure S7c).

We note that using the mode detector as incoupling device its efficiency drops to values similar than the dipole antenna. The difference in both cases we attribute to excitation by a single Gaussian mode, in contrast to emission in a non-Gaussian mode.

\section{Full transmission}

The full transmission shown in figure $3 \mathrm{~d}$ of the main manuscript is a multiplication of the three contributions: $\eta_{i n, i} \cdot \eta_{w g, i} \cdot \eta_{\text {det }, i}$, where $i$ stands for the case of symmetric or anti-symmetric mode. 

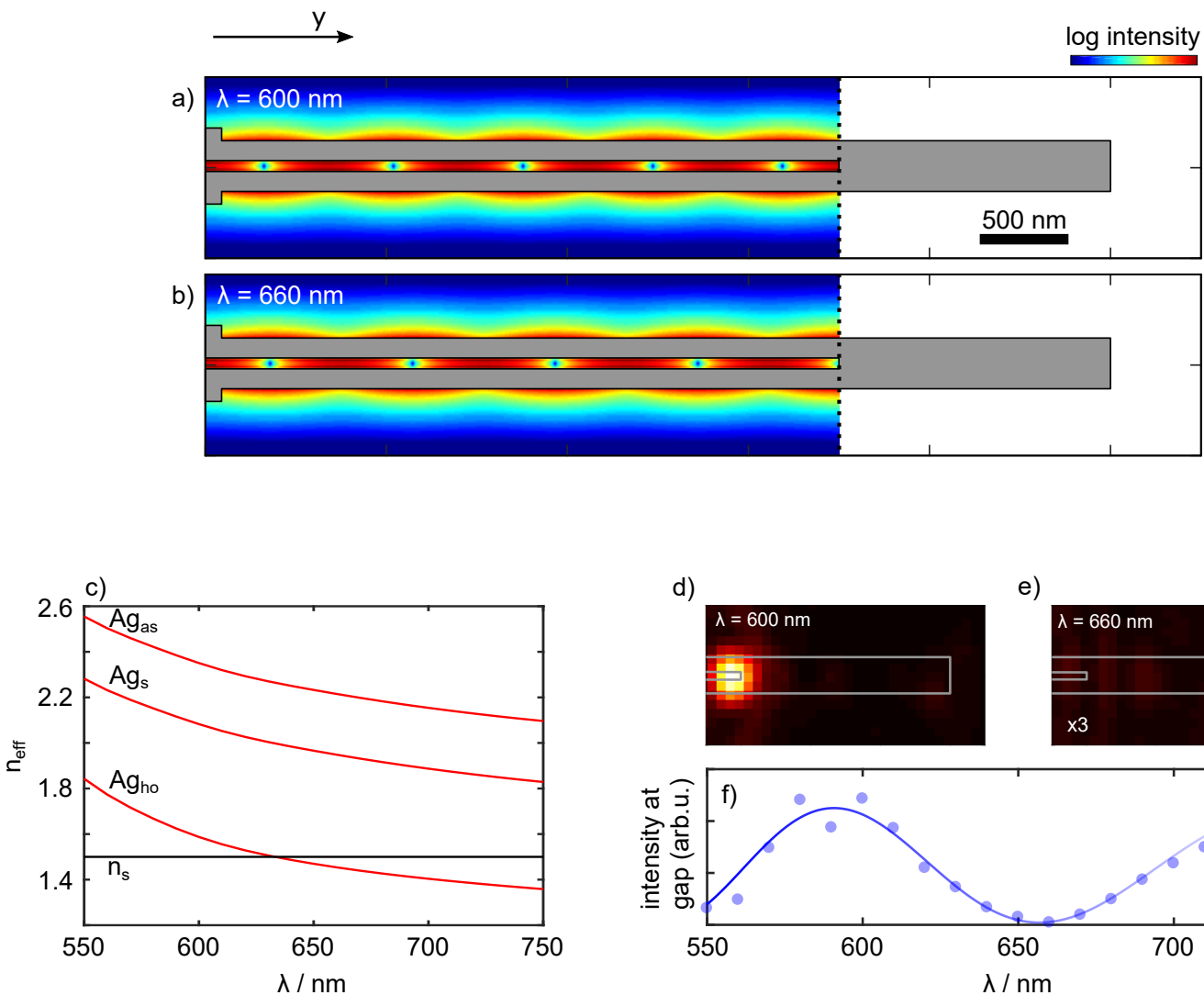

e)

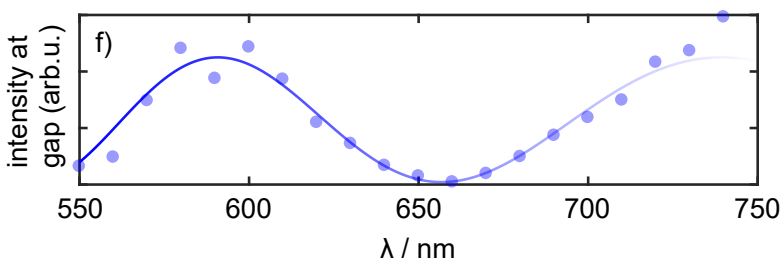

Figure S8: Field intensity (log scale) at $600 \mathrm{~nm}$ (a) and $660 \mathrm{~nm}$ (b) in a plane $20 \mathrm{~nm}$ above the substrate using a superposition of the anti-symmetric and the higher order mode. Details see text. The gray two-wire waveguide is overlaid for visualization of the gap termination position. (c) Simulated effective waveguide mode indices of the anti-symmetric (as), symmetric (s) and higher order mode (ho) as a function of wavelength $\lambda$ for the geometry shown in figure 1 of the main manuscript. (d) Experimental mode detector signal at $600 \mathrm{~nm}$ showing a strong single lobed emission only at the gap termination. (e) Experimental mode detector signal at $660 \mathrm{~nm}$ showing no emission at the gap termination and a two-lobed emission at the far end of the mode detector. (f) Simulated intensity at the gap termination as a function of wavelength (blue, solid) scaled to the experimental data (blue dots).

To calculate the beating pattern between the anti-symmetric and the higher order mode, we use the modal fields simulated by a 2D mode analysis at $620 \mathrm{~nm}$ (see figure 1 of the main manuscript). The fields are propagated along the two-wire waveguide (y-direction) with the propagation term $\exp \left(i 2 \pi / \lambda_{0} n_{e f f, i} y\right)$. Here the parameter $i$ distinguishes between the anti-symmetric and higherorder mode. A beating pattern, where the intensity oscillates between inside and outside the gap, results.

Changing the free space wavelength $\lambda_{0}$ shifts the beating pattern along the two-wire waveguide. At $600 \mathrm{~nm}$ the intensity is located inside the gap when the gap terminates (figure S8a), while at $660 \mathrm{~nm}$ the intensity vanishes inside the gap when it terminates (figure S8b).

In the modelling, we assume equal amplitues and no phase-shift between the modes at $y=0$ 
(antenna position) and fix the difference in mode indices between anti-symmetric (as) and higher order (ho) mode $\Delta \mathrm{n}=n_{\text {eff,as }}-n_{\text {eff,ho }}$ to 0.84 for simplicity. This latter $\Delta_{n}$ is a fitted value to match the experimental observations and is very close to the simulated difference of the $n_{\text {eff }}$ values (figure S8c). Together with the wavelength $\lambda_{0}, \Delta \mathrm{n}$ defines the beating periodicity: $\lambda_{0} / \Delta \mathrm{n}$. Propagation losses are neglected in the calculation, since the superposition of the two modes results in an average effective propagation length between the values of the separate modes, which we expect to be spectrally flat (c.f. figure 1 of the main manuscript).

The simulation behavior is consistent with experimental results, that show a strong emission at the gap termination at $600 \mathrm{~nm}$ (figure S8d), and no emission at $660 \mathrm{~nm}$ (figure S8e). In the latter case a two-lobed emission is observed at the far end of the mode detector which is characteristic for emission of the mode detector from a higher order mode. Thus, it can be distinguished from the single lobed emission in the case of the symmetric mode. We integrate the simulated intensity at the gap termination as a function of free space wavelength $\lambda_{0}$, yielding a spectral beat pattern (figure S8f, blue line). The result of this simple model fits very well to the spectral variation of the experimental data (dots in figure S8f). For higher wavelengths deviations set in, since the higher order mode gets more and more leaky and the transmission is dominated by the anti-symmetric mode alone. 

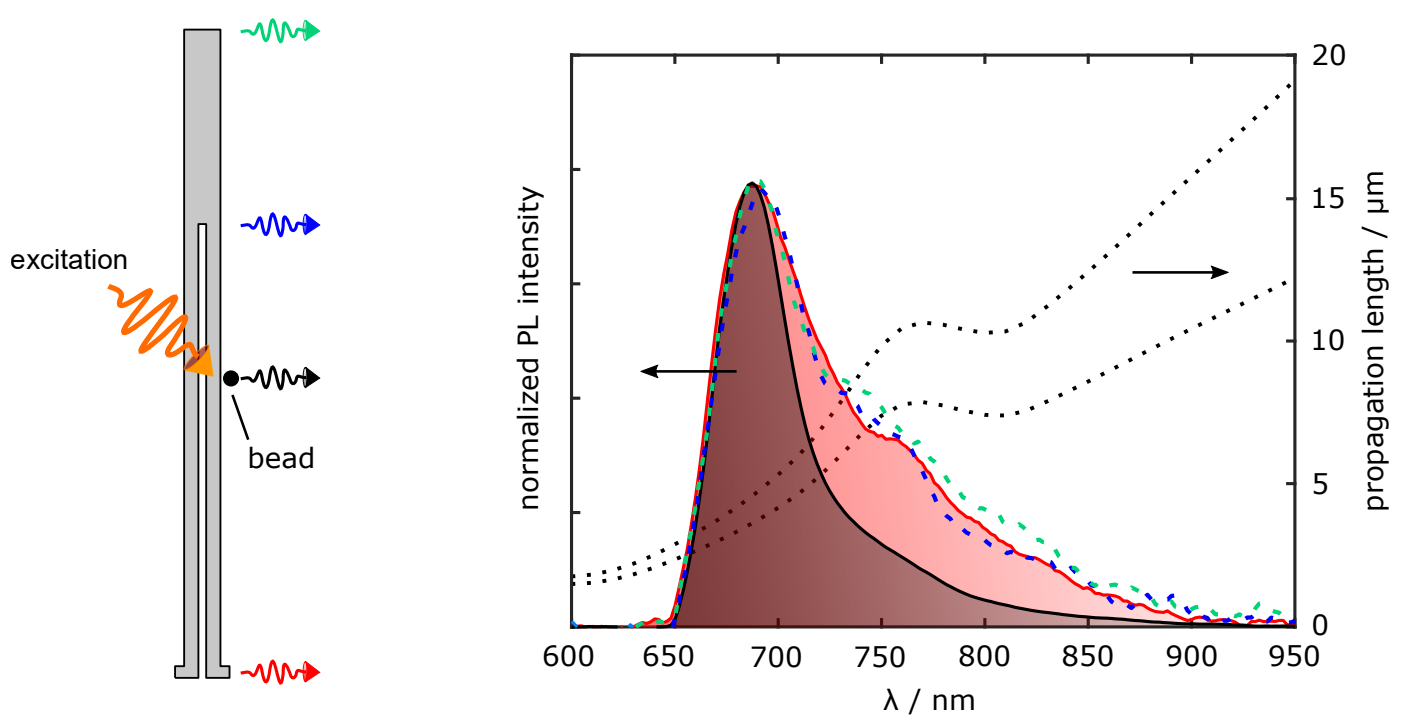

Figure S9: Normalized photoluminescence (PL) emission specta of a coupled dark red bead (solid black) upon excitation at a wavelength of $620 \mathrm{~nm}$ and of the guided emission detected at the antenna (solid red), near- (dashed blue) and far- (dashed green) end of the mode detector. For typical intensity ratios see manuscript. The red part of the guided emission is enhanced compared to the direct far-field emission of the bead. This effect is a consequence of the higher propagation lengths of the fundamental symmetric and anti-symmetric modes toward higher wavelengths (dotted black lines). 